\title{
AN INTEGRATED MICROCOMPUTER SYSTEM USING IMMOBILIZED CELLULAR ELECTRODES FOR DRUG SCREENING
}

\author{
Xiang-Ming Li*, Richard M. Schwartz*, Evangeline Y. Cesar $†$ and \\ HENRY Y. WANG* \\ * Department of Chemical Engineering, The University of Michigan; \\ and \\ † Process Biotechnology Inc., Ann Arbor, Michigan 48105, U.S.A. \\ (Received 26 May 1987; received for publication 5 January 1988)
}

\begin{abstract}
Biosensors based on immobilized microbial cells were developed for drug screening in our laboratory. Compared with the conventional methods such as diffusion and dilution tests, biosensors have been demonstrated to be superior in sensitivity and require much shorter screening time. An integrated microcomputer system has been developed for data acquisition, database management, and mode of action estimation, which automates the screening processes and reduces the labor requirements significantly.
\end{abstract}

Biosensor Automatic drug screening Data acquisition

Database management Expert system

\section{INTRODUCTION}

In search for anti-tumor and anti-microbial drugs, a great deal of effort has been spent in biological testing and screening. Conventional testing and screening methods, such as diffusion and dilution tests [1], are labor-intensive in both the testing procedures and subsequent data analyses. Biosensors have been suggested as a promising alternative for biological testing [2-4].

In our laboratory, biosensors with immobilized mammalian and microbial cells were developed to test the activities of anti-tumor and anti-microbial drugs $[5,6]$. The biosensor is consisted of two parts. One is the disposable immobilized cellular disk. The other is a dissolved oxygen (D.O.) electrode. The mammalian cells and microbial cells used in testing were immobilized in various matrices such as a filter paper disk coupled to the electrode as shown in Fig. 1. These cellular disks can be attached to the tip of the D.O. electrode just prior to testing. The biosensor assembly can then be put in a temperature controlled vessel containing drug and culture medium for testing. Filtered sterile air is bubbled through the medium to supply oxygen for the cells. The interaction between the cells and the drug can be detected indirectly by monitoring the oxygen uptake of the cells. When the cells grow, more oxygen will be consumed and less oxygen will diffuse into the D.O. electrode. When the cells are inhibited or killed by the drug, more oxygen can diffuse through the cellular disk and reach the electrode surface, resulting in a higher current output of the electrode when compared to the control. Unlike the diffusion and dilution tests which provide only end points results, biosensor response curves reveal drug and cell interaction during the entire testing period. The response curve patterns of the biosensors can be used to evaluate various drugs as demonstrated by our experimental results.

In order to reduce the labor intensive effort required in testing and data analyses, we have developed in our laboratories an integrated microcomputer system for data acquisition, data processing, database management, and mode of action estimation. The biosensor output can 


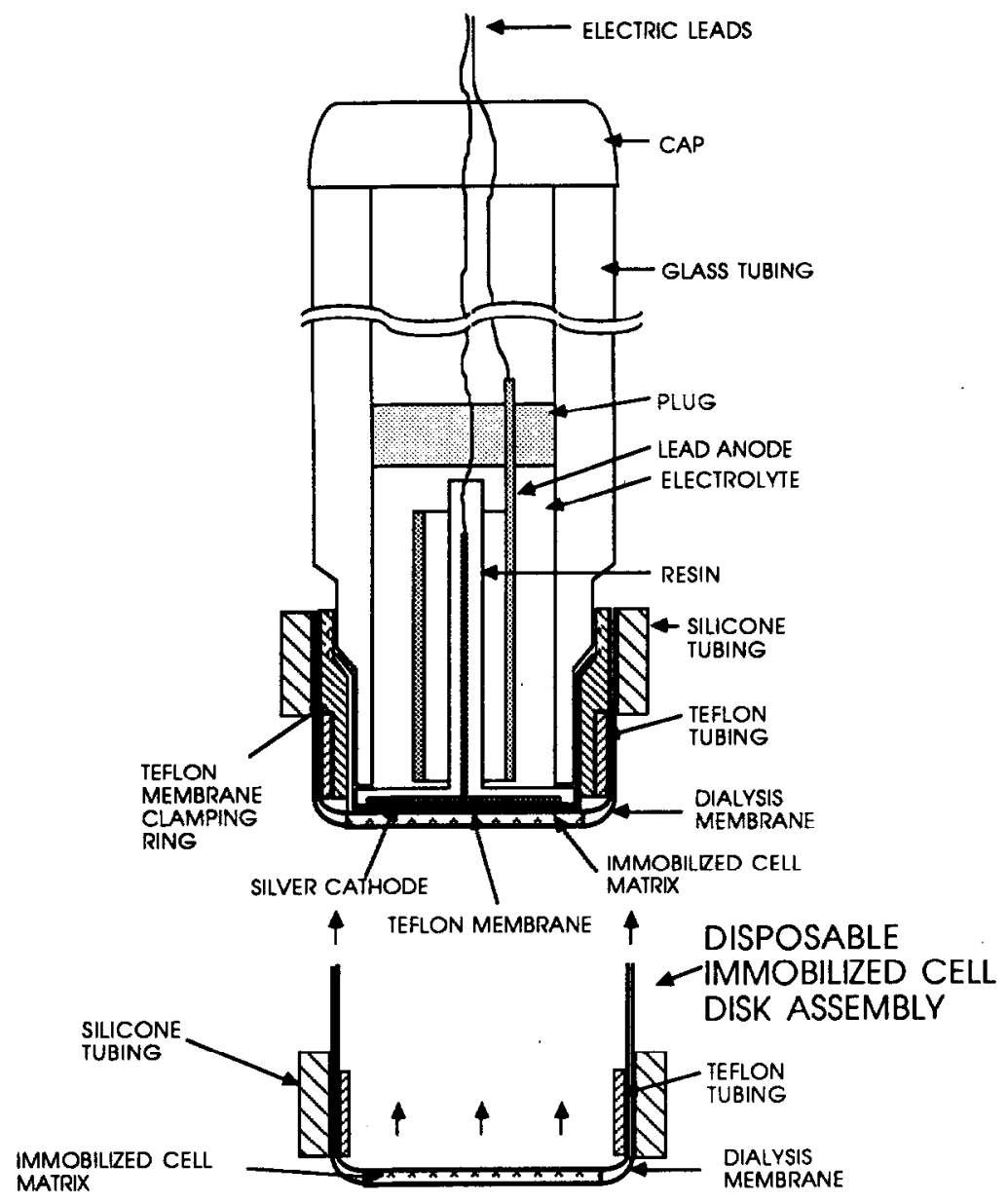

Fig. 1. Schematic diagram of biosensor.

be stored in a pre-formatted database without off-line processing. These records can be retrieved from the database using several key words and then compared with old and new records in tabulated or graphical forms. A prototype expert system was also developed for consultation support to evaluate various drugs and chemicals.

\section{EXPERIMENTAL RESULTS}

Immobilized $E$. coli and yeast cell disks were used in our initial experimental models. Various antibiotics (or drugs) with well known modes of action, such as ampicillin, tetracycline, nystatin, and cychloheximide were employed as model drugs for comparison and demonstration purposes: The experimental setup and procedures are essentially the same as those described in earlier published results from this laboratory $[5,6]$.

Some typical response curves of the biosensors are displayed in Figs 2-5. Figure 2 shows the response curves of the biosensor with immobilized $E$. coli cells at different ampicillin concentrations. The initial cell concentration in the cellular disks is around $3.3 \times 10^{7}$ cells $/ \mathrm{ml}$. When the drug concentrations greater than $10 \mu \mathrm{g} / \mathrm{ml}$ are used, the current output of the biosensor shows a similar pattern, e.g. the biosensor current output gradually decrease initially, and then gradually increase afterward. This pattern can be related to the mode of action of ampicillin on $E$. coli. Ampicillin is a drug which inhibits the cell wall synthesis of $E$. coli [7] thus causing cell wall leakage. A reduction of the current output during the first part 
of the curve correlates to initial cell growth (and therefore more oxygen uptake). The rise during the second part of the curve suggests the growing cells lose their viability (and therefore less oxygen uptake) as the drug exerts its action on the growing cells and causes

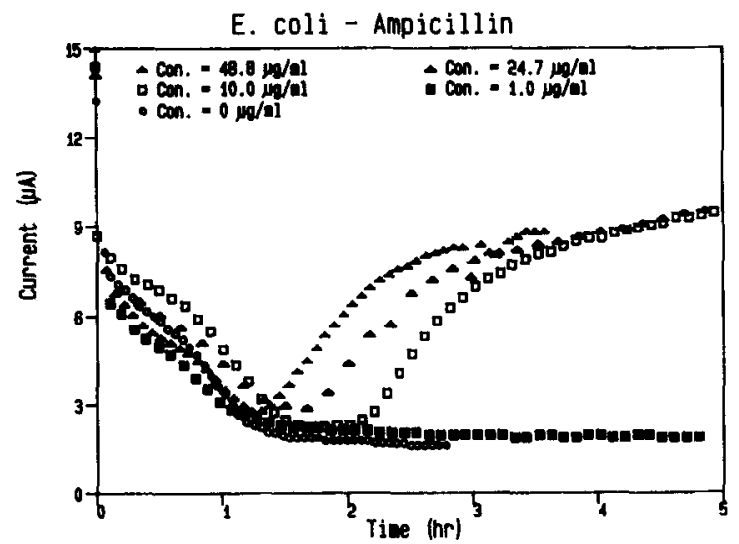

Fig. 2. Effect of different concentrations of ampicillin on the $E$. coli biosensor current output. Initial cell concentration $=3.3 \times 10^{7} \mathrm{cell} / \mathrm{ml}$, tested in growth medium, temperature $=30^{\circ} \mathrm{C}$, current output of biosensor with dead cells $=13 \mu \mathrm{A}$.

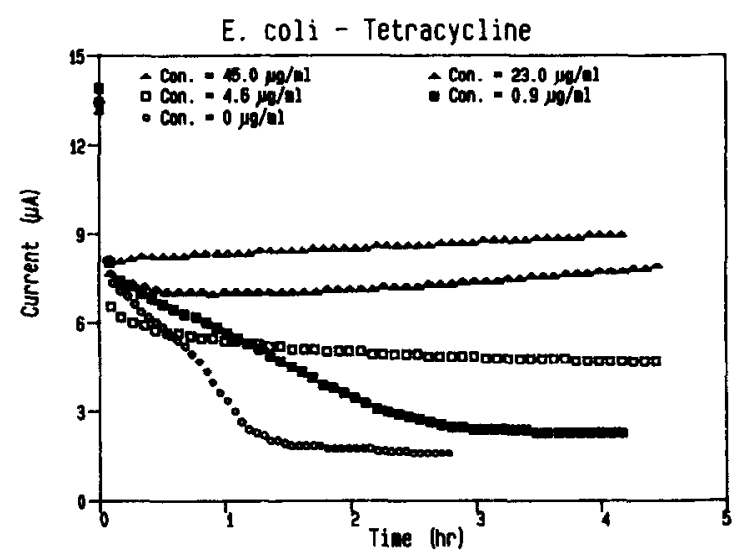

Fig. 3. Effect of different concentrations of tetracycline on the $E$. coli biosensor current output. Initial cell concentration $=3.3 \times 10^{7} \mathrm{cell} / \mathrm{ml}$, tested in growth medium, temperature $=30^{\circ} \mathrm{C}$, current output of biosensor with dead cells $=13 \mu \mathrm{A}$.

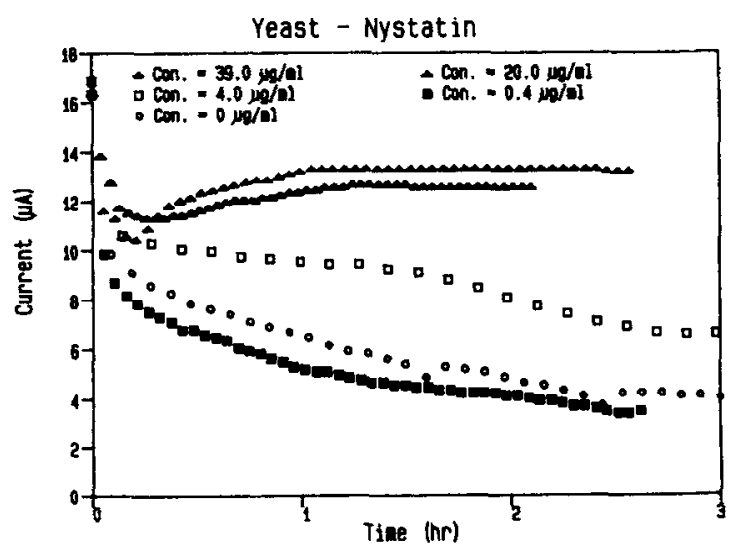

Fig. 4. Effect of different concentrations of nystatin on the yeast biosensor current output. Initial cell concentration $=2.7 \times 10^{8} \mathrm{cell} / \mathrm{ml}$, tested in growth medium, temperature $=30^{\circ} \mathrm{C}$, current output of biosensor with dead cells $=13 \mu \mathrm{A}$. 


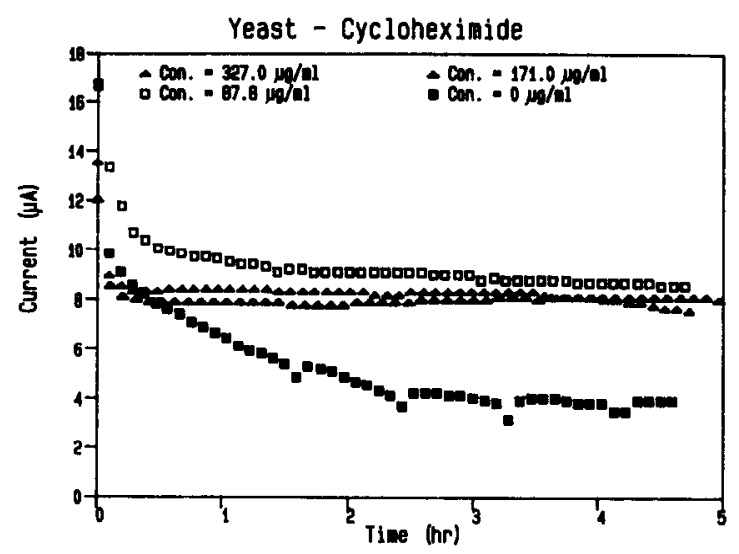

Fig. 5. Effect of different concentrations of cycloheximide on the yeast biosensor current output. Initial cell concentration $=2.7 \times 10^{8} \mathrm{cell} / \mathrm{ml}$, tested in growth medium, temperature $=30^{\circ} \mathrm{C}$, current output of biosensor with dead cells $=13 \mu \mathrm{A}$.

serious damage to the cells. At a drug concentration of $1 \mu \mathrm{g} / \mathrm{ml}$, the response curve is similar to the control $(0 \mu \mathrm{g} / \mathrm{ml})$. This indicates that the minimum inhibitory concentration (MIC) is between 1 and $10 \mu \mathrm{g} / \mathrm{ml}$, which agrees with previous published results $(8 \mu \mathrm{g} / \mathrm{ml})$ [7]. More exact MIC concentration can be determined using narrower ampicillin concentrations. Figure 3 shows the effect of different concentrations of tetracycline on the output of biosensor with immobilized $E$. coli cells. Tetracycline inhibits protein synthesis of bacterial cells [8], and can be shown to cause a complete different current output pattern of the biosensor with immobilized $E$. coli when compared to ampicillin. The current output remains nearly unchanged when the drug concentration is greater than $4.6 \mu \mathrm{g} / \mathrm{ml}$, suggesting the $E$. coli is growth inhibited. The MIC of tetracycline should be between 4.6 and $0.9 \mu \mathrm{g} / \mathrm{ml}$ as indicated from Fig. 3, which is in agreement with the previous MIC data $(4 \mu \mathrm{g} / \mathrm{ml})$ in the literature [7].

Figures 5 and 6 show the effects of different concentrations of nystatin and cycloheximide on the output of biosensors using immobilized yeast cells. The initial cell concentration in the cell disks is $2.7 \times 10^{8}$ cells $/ \mathrm{ml}$. The mode of action of nystatin is on membrane function, and cycloheximide is on protein synthesis [8]. Again, drugs with different modes of actions show different effects on the biosensor output. By using multiple cellular disks with different cells, an efficient screening test can thus be developed.

Based on these experimental results, we demonstrated the feasibility of using biosensors for testing the activity of various drugs such as susceptibility of various microbial cells to antimicrobial agents in a relatively short period of time $(2-5 \mathrm{hr}$.). The correlation between the response patterns of the biosensor and the mode of action of the drugs also demonstrates the possibility of using a pattern recognition technique to partially explain the mode of action of various drugs and chemicals based on the electrode response curves. Furthermore, biosensors show significant advantages in sensitivity when compared to conventional methods [1], which often required more then one day to complete the testing. Using appropriate initial cell concentrations $\left(1 \times 10^{7}\right.$ to $1 \times 10^{9}$ cells $\left./ \mathrm{ml}\right)$, the change of cellular oxygen uptake caused by various immobilized procaryotic and eucaryotic cells can be detected continuously. Another advantage worth noting is the biosensor output, which is an electrical signal, can be readily processed using a computer automatically.

Using biosensors to screen potential antimicrobial/antitumor agents can reduce the time required for each assay, however it cannot reduce the number of assays in a screening program. Comparison of many different assay results is needed to elucidate a meaningful conclusion. When large numbers of assays are carried out, searching and comparing data in various forms becomes very labor intensive. Manual data reduction, book keeping and plotting work will significantly slow down the screening program. Automation of data collection, book keeping, and data analysis becomes an important requirement for a large scale biological testing and screening program. 
In the last few years, the capacity and speed of microcomputers have increased several fold and the price/performance ratio has dropped significantly. Development of an integrated microcomputer system which includes the functions of automatic data acquisition, database management, and expert system for consultation support, becomes very beneficial for using biosensor in large scale screening and testing. The developed biosensor based integrated microcomputer system addresses these concerns.

\section{GENERAL FEATURE OF THE SYSTEM HARDWARE AND SOFTWARE}

An integrated microcomputer system for biosensing was developed recently in our laboratory. The hardware and software of this system are displayed schematically in Fig. 6. We chose a COMPAQ portable II model 4 microcomputer (COMPAQ Computer Corporation, Houston, Texas) with 8-MHZ 80286 CPU, 640 KB RAM, 20 MB HARD DRIVE, $360 \mathrm{~KB}$ FLOPPY DRIVE, as our basic unit. This system can be adapted to any IBM PC XT or AT, or compatible microcomputer with $512 \mathrm{~KB}$ RAM and a hard disk drive. The data acquisition unit consists of a DASCON-1 multifunction analog/digital I/O expansion board and a STA-01 screw termination board (MetraByte Corporation, Stoughton, Massachusetts). The biosensor output is connected to the DASCON-1 board through the STA-01 board.

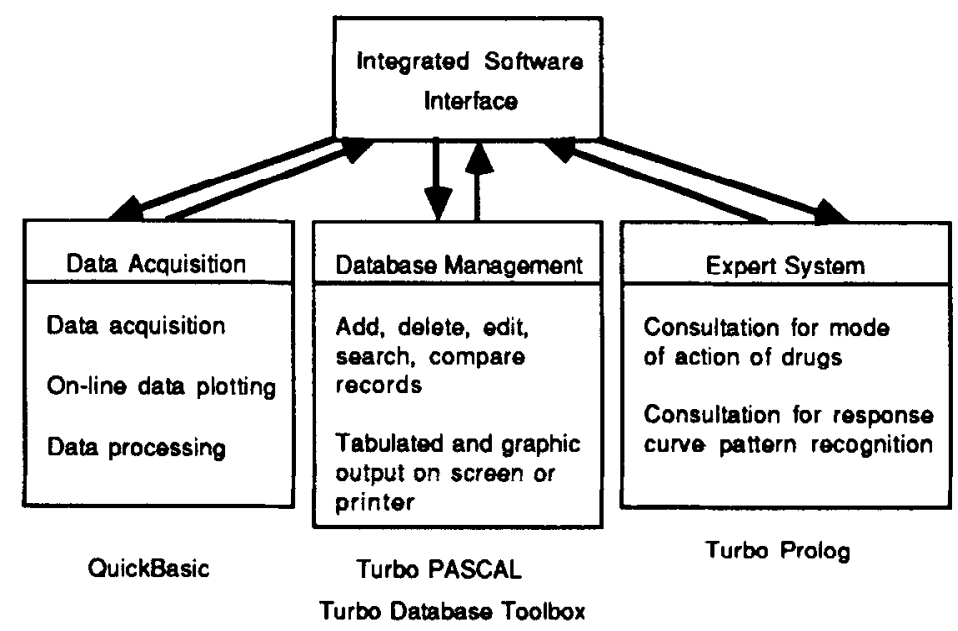

Integrated software structure

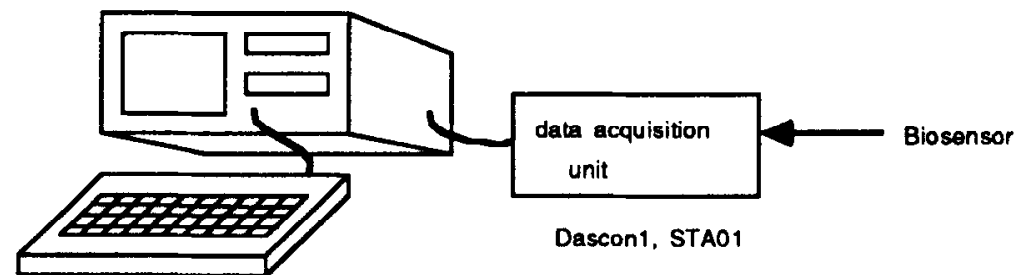

Compaq Portable II Model 4 Microcomputer

Hardware

Fig. 6. Computer software and hardware of the system. 
The software we developed is comprised of three parts. These are data acquisition, database management, and expert system, as shown in Fig. 6. The interface and the data acquisition parts are written in QuickBASIC (Microsoft Corporation, Redmond, Washington). QuickBASIC is an extension of the compiled BASIC language. It supports structure and modular programing. The I/O driver routine for DASCON-1, a machine language routine provided by MetraByte, can be placed in a user library and called from QuickBASIC for data acquisition. The SHELL statement supported by QuickBASIC enables us to execute other parts of the programs written in different languages from the interface. Disk files are used for communication between different parts of the program written in different languages. The database management program is written in Turbo PASCAL (Borland International, Inc., Scotts Valley, CA) for the numerous data structures supported by this language. Some of the procedures in the Turbo Database Toolbox (Borland International, Inc., Scotts Valley, CA) are employed in the database management part of the program. The expert system is written in Turbo Prolog (Borland International, Inc., Scotts Valley, CA). Turbo Prolog is an object-oriented language for application that involves formal or symbolic reasoning [9]. With Prolog, the user defines a goal or an objective, and the computer can find both the procedure and the solution based on the facts and the relationships among these facts encoded in the program. The compiled Turbo Prolog programs execute faster than any other Prolog programs on the market for the IBM PC and compatible computers [9]. Based on our experience, Turbo Prolog is a good language for developing small and mid-size expert systems for microcomputer.

The software for biosensing is a menu-driven user-friendly program. All the functions can be chosen from menus and can be executed in an interactive way. Instructions and warnings are provided wherever they are necessary. People do not need any knowledge in programming to use this software. More details of the software will be presented in the following sections.

\section{DATA ACQUISITION}

The data acquisition module of the software provides all the functions needed for data acquisition and data processing. The following functions can be selected from the main menu: (1) data acquisition; (2) plot data; (3) take more data; (4) read old data from disk file; (5) data processing; (6) database management; (7) expert system; (8) quit. The data acquisition starts by asking the user to enter the time interval between data points and the total sampling time for reading the output of the biosensors into the computer memory. The time interval and total sampling time can be changed at any time during data acquisition. Whenever data are read from the biosensor, they are also displayed on the screen and stored in a disk file. During data acquisition, pressing function key F1 can display an interrupt menu on the screen. Several functions can be chosen from this menu. These functions are: (1) change time interval, total sampling time, and continue data logging; (2) stop taking data and go back to main menu; (3) plot previous data; (4) continue data logging; (5) clear previous data and start again. When the total sampling time is reached, the data logging will stop and the main menu will reappear on the screen. All the functions in the main menu can be selected repeatedly by the user. If the "plot data" function is selected, the biosensor response curve will be plotted on the screen. If the "take more data" function is selected, the data acquisition will continue according to the new time interval and the new total sampling time entered by the user. When the "data processing" function is selected, a data processing menu will appear on the screen. The following functions can be selected from this menu: (1) plot data; (2) select new time range; (3) find maximum gradient of the response curve, and the maximum and minimum current output. The function of selecting new time range enables one to choose the data in a specified time range to be stored in the database, and discards the rest of the data. The data at the beginning of new time range specified will be shifted to time zero and the time frame of the rest of the data will also be shifted accordingly. After the data acquisition and data processing, the data can be stored in the database using the function of database management. If the data has more than fifty points, the data will be interpolated into fifty 
points and stored in a data buffer. These data points will later be read into the database by the database management program.

\section{DATABASE MANAGEMENT}

The database management module is developed specifically for managing the data acquired in bioscreening. Three main functions can be selected from the main menu of the database management module for book keeping and data comparison. They are: (1) add data records; (2) list data records; (3) find data records.

The data structure of each data record is displayed in Fig. 7. The basic data records consist of 12 elements. They are: (1) record number; (2) entry date; (3) type of sensor; (4) cell line; (5) chemical (drug); (6) mode of action; (7) curve shape; (8) data file name for the response curve data; (9) cell concentration; (10) chemical concentration; (11) testing temperature; (12) remarks. All the elements, except record number and data file name for the response curve data, are entered by the user. A unique record number and a unique data file name for the response curve data are generated by the database management program. The data of the response curve, including time and current output, are transferred automatically from the data buffer to the data file using the file name stored in the data record when the new data

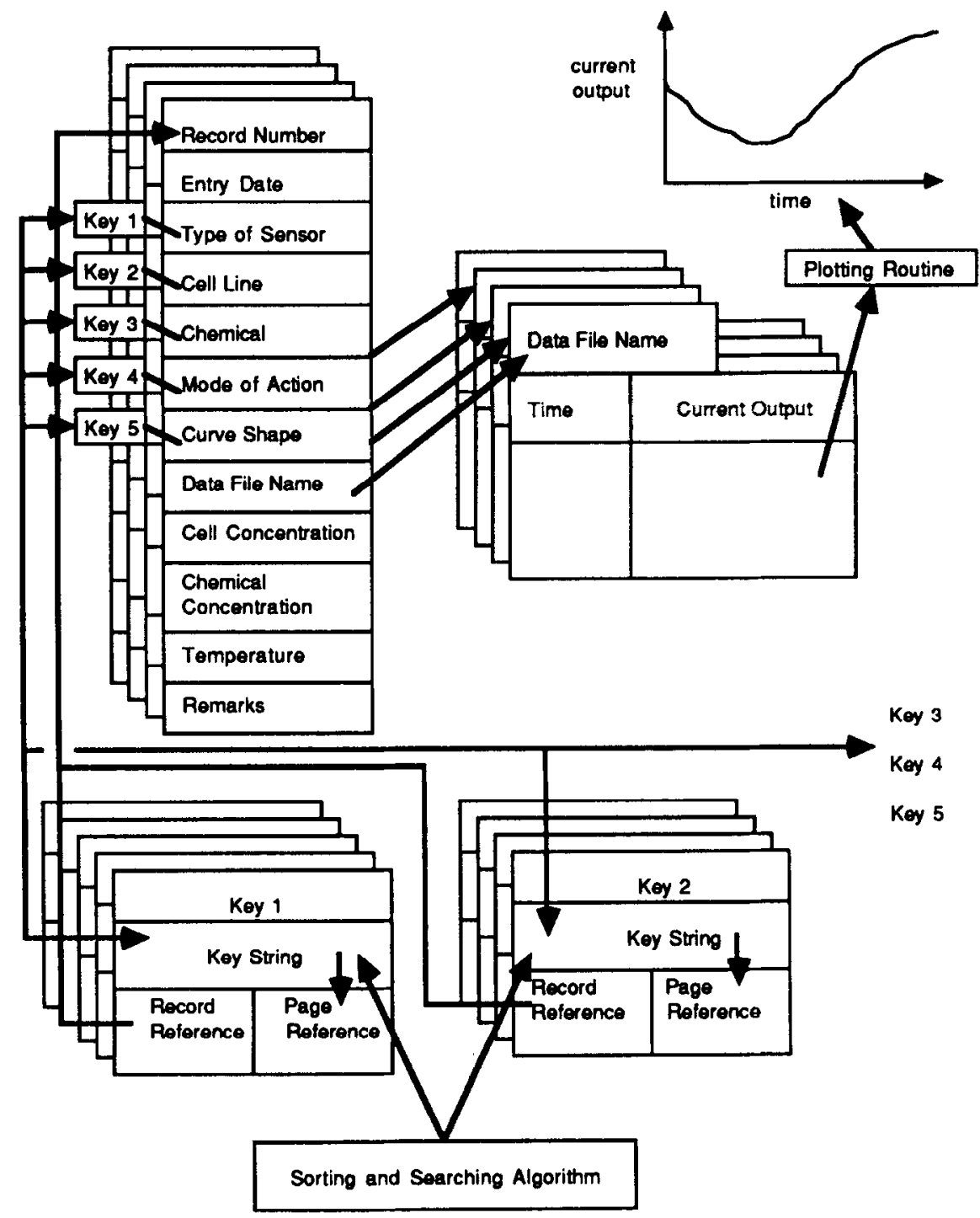

Fig. 7. Data structure of the biosensor database. 
and added to the database. The data file name is the link between each data record and its response curve data. Since each data record has a length of 405 bytes, sorting and searching the specific elements in the data records will be slow. In order to reduce the sorting and searching time, five record elements, e.g. type of sensor, cell line, chemical, mode of action, and curve shape, are treated as key element, and put into five key files. Each key file contains one of the key elements for all the records. These key files with a B-tree structure can be sorted and searched efficiently $[10,11]$. Any combination of these keys (from one to five) can be selected as the key words to search all the data records in the database which have the key elements that can match these keys.

Two functions of the database management module can be used to retrieve the data records. The first is "List". By using this function, the data records can be listed one by one, sorted by one of the key elements specified by the user. These data records and the response curves can be outputed either to the computer screen or to the printer. The other function for retrieving the data records is "Find". After the user chooses this function, the computer provides the prompts for entering the key words and then individually displays the data records which match these key words. When the data record is displayed on the computer screen in the tabulated form, a select option window is also displayed on the bottom of the screen. The options in the select window include: (1) print record on printer; (2) plot response curve on screen; (3) select record as basic or compared record for comparison; (4) plot the response curve of the current record with any other response curve; (5) edit the record; (6) delete the record.

With this database management module, any data record can be retrieved and compared with each other in tabulated and graphical forms using a few key strokes.

\section{EXPERT SYSTEM}

The purpose of the prototype expert system we developed is to help people evaluate and prescreen various drugs and chemicals. It has two functions: (1) consultation for mode of action of a drug; (2) consultation for response curve pattern recognition. The consultation for the mode of action of a drug is based on a knowledge base encoded in the expert system which includes more than two hundred drugs with known mode of actions acquired from literature $[12,13]$. The consultation for response curve pattern recognition is based on a knowledge base representing the correlations between the response curve patterns and the modes of actions of the drugs we obtained from various experiments. Figures 2-5 showed some of the examples of the biosensor response curves for various antibiotics having different modes of actions against different kind of cells. These response curve patterns can be related to the mode of action qualitatively. For example, the E. coli biosensor response curves for ampicillin are $\mathrm{V}$ shaped which can be related to the possible cell wall synthesis inhibition, while the $E$. coli biosensor response curves for tetracyclinc are ncarly a straight line which can be related to possible protein synthesis inhibition. In the case where the drug concentration is higher than the minimum inhibition concentration (MIC), the response curves of a specific biosensor may have similar response patterns. However, please keep in mind that now only the mode of action of the drug, but also other factors, such as the concentration and the diffusivity of the drug, may also affect the shape of the response curves. Thus, this correlation between the drug mechanism and the response curve pattern can only be a qualitative one. We cannot "calculate" the mode of action based on the response curve. Instead, we try to simulate the human reasoning processes in this expert system to evaluate the mode of action of various drugs based on comparison of the acquired response curve pattern with the patterns of those drugs with well known modes of actions. These response curve patterns can be encoded in the expert system as rules and facts. During consultation, the user tells the expert system how the response curve obtained looks like by answering the questions that are raised by the expert system. Then the program will search the knowledge base to find the "match". If one or more matches are found, the program can tell the user the possible (not exclusive) modes of actions of the drug. 


\title{
SUMMAR Y
}

Experimental results presented in this paper and the previous paper $[5,6]$ demonstrate that biosensors using detachable cellular disks are promising devices for antimicrobial/ antitumor screening. They showed higher sensitivity and require much shorter assay time compared to the dilution and diffusion tests. Unlike the conventional methods which provide only end point results, biosensors reveal continuous chemical and cell interaction during the entire screening period. Our experimental results also demonstrated interesting correlations between the response curve patterns and the modes of actions for various drugs. In order to effectively utilize the significant amount of information generated during screening, an integrated microcomputer system has been developed for data acquisition, database management, and mode of action estimation. The biosensor output can be stored in a pre-formatted database without off-line processing. The data records can be retrieved from the database using several key words and these records can be displayed and compared in either tabulated or graphical forms for evaluation. In conjunction with this system, a prototype expert system was developed for consultation support to evaluate various drugs or chemicals. The integrated microcomputer system provides a powerful tool for using biosensors in biological testing and screening. This system significantly reduced the labor requirements for data acquisition, data processing, and data management. It automated the biosensing processes and reduced the possible human errors in these processes.

\section{REFERENCES}

1. E. H. Lennette, A. Balows, W. J. Hausler, Jr and J. P. Truant, Manual of Clinical Microbiology, 3rd Edn, Chapters 41-43. American Society for Microbiology, Washington, D.C. (1980).

2. C. R. Lowe, An introduction to the concepts and technology of biosensors, Biosensors 1, 3-16 (1985).

3. K. Matsumoto, H. Seijo, T. Watanabe, I. Karube, I. Soath and S. Suzuki, Immobilized whole cell-based flow-type sensor for cephalosporins, Analytica Chim. Acta, 105, 429-432 (1979).

4. M. Hikuma, T. Kubo, T. Yasuda, I. Karube and S. Suzuki, Amperometric determination of acetic acid with immobilized Trichosporon brassicae, Analytica Chim, Acta, 109, 33-38 (1979).

5. B. S. Liang, X. M. Li and H. Y. Wang, Cellular electrode for antitumor drug screening, Biotechnology Progress 2, 187-191 (1986).

6. X. M. Li, B. S. Liang and H. Y. Wang. Computer aided analysis for biosensing and screening, Bitech. Bioeng. 31, 250-256 (1988).

7. T. D. Brock, D. W. Smith and M. T. Madigan, Biology of Microorganisms, 4th Edn, p. 516. Prentice-Hall, New Jersey (1984).

8. D. Gottlieb and P. D. Shaw, Antibiotics, Vol. 1, Mechanism of Action, p. 764. Springer, New York (1967).

9. C. Townsend, Introduction to Turbo Prolog, SYBEX, Berkeley, (1987).

10. Owner's Handbook of Turbo Database Toolbox, Borland, pp. 131-145 (1985)

11. E. Horowitz, Fundamentals of Data Structure. Pitman (1976).

12. D. Gottlied and P. D. Shaw, Antibiotics, Vol. 1, Mechanism of Action. Springer, New York (1967).

13. L. P. Garrod, H. P. Lambert and F. O'Grady, Antibiotic and Chemotherapy. Churchill Livingstone, Edinburgh (1981).

\begin{abstract}
About the Author - Evangeline Yap Cesar received her Ph.D. degree in Pharmaceutical Chemistry from the University of Michigan in 1978. She joined the Medical Research Division of American the Research Institute of Chemical Engineering of South China Institute of Technology, Quangzhou, China. After receiving his $\mathrm{Ph}$.D. he spent two years as a Postdoctoral Fellow in Biotechnology at the University of Michigan. He is currently a Professor at South China Institute of Technology. His research activities center around computer aided chemical and biochemical engineering analyses with particular emphasis on biosensing and bioinstrumentation.
\end{abstract}

About the Author-Richard M. Schwartz received his B.S. in Chemical Engineering at the University of Michigan in 1981. From 1981-1986, he was a staff engineer at Eli Lilly and Company at Indianapolis, Indiana. Currently, he is a graduate student pursuing his Ph.D. at the University of Michigan. 
About the Author-Evangeline YaP Cesar received her Ph.D. degree in Pharmaceutical Chemistry from the University of Michigan in 1978. She joined the Medical Research Division of American Cyanamid Co. at Pearl River, New York. Her primary research activities had centered on the stabilities and the optimization of drug delivery from the different pharmaceutical dosage formulations. She joined Process Biotechnology, Inc. in Ann Arbor and is the Principal Investigator of several active SBIR projects funded by NIH and NSF.

About the Author-Henry Y. Wang received his B.S. in Chemical Engineering from Iowa State University in 1972 and S.M. and Ph.D. degrees in Biochemical Engineering from M.I.T. in 1974 and 1977 respectively. After several years of industrial experience at Merck \& Co. Inc. and ScheringPlough, Co., he joined the Department of Chemical Engineering at the University of Michigan. He is currently Associate Professor of Biochemical Engineering in the same institution. He has a joint appointment as Distinguished Senior Scientist at the Michigan Biotechnology Institute. His research interests include biosensing and bioscreening, cell cultivation technology and bioseparation processes with emphasis on computer application. He is the author of numerous scientific publications. 